\title{
Comparison of the critical shoulder angle in radiographs and computed tomography
}

\author{
Bouaicha, Samy ; Ehrmann, Christine ; Slankamenac, Ksenija ; Regan, William D ; Moor, Beat K
}

\begin{abstract}
The critical shoulder angle (CSA) is an indicator of degenerative shoulder pathologies. CSAs above $35^{\circ}$ are associated with degenerative rotator cuff disease, whereas values below $30^{\circ}$ are common in osteoarthritis of the glenohumeral joint. Measurements are usually performed on radiographs; however, no data have been reported regarding the reliability of CT scan measurements between different readers or the reproducibility of measurements from radiographs to CT scans. The purpose of our study was to clarify whether CSA measurements on radiographs and CT scans of the same patients show similar values. Materials and methods: CSA measurements of 60 shoulders (59 patients) were performed on radiographs and multiplanar reconstructions of corresponding CT scans. Inter-reader reliability and inter-method correlation were calculated. Results: The mean discrepancy between readers was only $0.2^{\circ}\left(\mathrm{SD} \pm 1.0^{\circ}\right)$ on radiographs. CT scan measurements showed a mean discrepancy of $0.3^{\circ}\left(\mathrm{SD} \pm 1.2^{\circ}\right)$. The inter-reader reliability was 0.993 for radiographs and 0.989 for CT scans. There was a very strong inter-method correlation between the CSA measured on radiographs and CT scans (Spearman's rho $=0.974$ ). The mean differences between angles on radiographs and $\mathrm{CT}$ measurements were $-0.05^{\circ}\left(\mathrm{SD} \pm 1.2^{\circ}\right)$ and $0.1^{\circ}$ $\left(\mathrm{SD} \pm 1.2^{\circ}\right)$, respectively. Conclusion: Measurements of the CSA on anterior-posterior radiographs and CT scans are highly correlated, and inter-modality differences are negligible.
\end{abstract}

DOI: https://doi.org/10.1007/s00256-014-1888-4

Posted at the Zurich Open Repository and Archive, University of Zurich

ZORA URL: https://doi.org/10.5167/uzh-172002

Journal Article

Published Version

Originally published at:

Bouaicha, Samy; Ehrmann, Christine; Slankamenac, Ksenija; Regan, William D; Moor, Beat K (2014). Comparison of the critical shoulder angle in radiographs and computed tomography. Skeletal Radiology, 43(8):1053-1056.

DOI: https://doi.org/10.1007/s00256-014-1888-4 


\title{
Comparison of the critical shoulder angle in radiographs and computed tomography
}

\author{
Samy Bouaicha $\cdot$ Christine Ehrmann • \\ Ksenija Slankamenac • William D. Regan • Beat K. Moor
}

Received: 26 January 2014 / Revised: 26 March 2014 / Accepted: 30 March 2014 /Published online: 18 April 2014

(C) ISS 2014

\begin{abstract}
Objective The critical shoulder angle (CSA) is an indicator of degenerative shoulder pathologies. CSAs above $35^{\circ}$ are associated with degenerative rotator cuff disease, whereas values below $30^{\circ}$ are common in osteoarthritis of the glenohumeral joint. Measurements are usually performed on radiographs; however, no data have been reported regarding the reliability of CT scan measurements between different readers or the reproducibility of measurements from radiographs to $\mathrm{CT}$ scans. The purpose of our study was to clarify whether CSA measurements on radiographs and CT scans of the same patients show similar values.

Materials and methods CSA measurements of 60 shoulders (59 patients) were performed on radiographs and multiplanar reconstructions of corresponding CT scans. Inter-reader reliability and inter-method correlation were calculated.

Results The mean discrepancy between readers was only $0.2^{\circ}$ $\left(\mathrm{SD} \pm 1.0^{\circ}\right)$ on radiographs. CT scan measurements showed a mean discrepancy of $0.3^{\circ}\left(\mathrm{SD} \pm 1.2^{\circ}\right)$. The inter-reader reliability was 0.993 for radiographs and 0.989 for CT scans. There was a very strong inter-method correlation between the CSA measured on radiographs and CT scans (Spearman's
\end{abstract}

S. Bouaicha $\cdot$ W. D. Regan

Divison of Arthroscopic, Reconstructive Surgery and Joint

Preservation, Department of Orthopaedics, University of British

Columbia, Vancouver, Canada

S. Bouaicha $(\bowtie) \cdot K$. Slankamenac

Division of Trauma, Zurich University Hospital, Raemistrasse 100, 8091 Zurich, Switzerland

e-mail: samy.bouaicha@usz.ch

C. Ehrmann

Department of Radiology, University of British Columbia,

Vancouver, Canada

B. K. Moor

Department of Orthopaedics, Insel University Hospital, Berne, Switzerland rho $=0.974)$. The mean differences between angles on radiographs and $\mathrm{CT}$ measurements were $-0.05^{\circ}\left(\mathrm{SD} \pm 1.2^{\circ}\right)$ and $0.1^{\circ}\left(\mathrm{SD} \pm 1.2^{\circ}\right)$, respectively.

Conclusion Measurements of the CSA on anterior-posterior radiographs and $\mathrm{CT}$ scans are highly correlated, and intermodality differences are negligible.

Keywords Critical shoulder angle $\cdot$ Radiographs $\cdot$ Computed tomography

\section{Introduction}

The critical shoulder angle (CSA) is a new radiographic measurement tool that may indicate the presence of degenerative shoulder pathologies. This parameter revealed not only an association with degenerative rotator cuff tears but also with the presence of glenohumeral osteoarthritis. While a CSA greater than $35^{\circ}$ was correlated with degenerative rotator cuff tears, a CSA below $30^{\circ}$ was common in osteoarthritis of the glenohumeral joint $[1,2]$. The CSA measurement is usually performed on true anterior-posterior radiographs of the shoulder to determine the angle between the glenoid plane (superior glenoid rim to inferior glenoid rim) and the gleno-acromial plane (inferior glenoid rim to the most lateral extent of the acromion) [1]. Although the reliability of CSA measurements on radiographs has been proven (even on projections of a scapular position altered by up to $20^{\circ}$ [1]) there is no information regarding the measurement of the CSA on cross-sectional imaging, such as CT scans. Because CSA measurements are based on defined bony landmarks, it remains unclear if the measured angles differ between cumulative radiographs and uniplanar CT scans. In the present study, we aimed to determine the degree of correlation between CSA measurements on CT scans and radiographs and to determine if CSA measurements on $\mathrm{CT}$ scans are reliable between different readers. 


\section{Materials and methods}

Patients

A consecutive selection of anterior-posterior radiographs and corresponding CT scans of 60 shoulders (59 patients) were extracted from the hospital radiological database and retrospectively analysed. Patients with different clinical shoulder pathologies including instability, osteoarthritis and rotator cuff pathologies were included. Only complete data sets with sufficient image quality were eligible for the study. Radiographs and CT scans were obtained within a period of 12 months in every patient. Patients with bony deformities or significant wear (loss of glenoid contour) as well as patients with fractures of the glenoid, scapula spine or acromion were excluded. Data sets were anonymized after selection for further analysis.

All radiographs and CT scans were analysed by two independent readers, one orthopaedic surgeon (S.B.) and one fellowship-trained musculoskeletal radiologist (C.E.). Measurements of both radiographs and CT scans were performed on PACS stations in the radiology department. This study was conducted according to the medical-ethical guidelines of our institution for retrospective data analysis.

\section{Measurements on radiographs}

As previously described, the CSA was measured on true anterior-posterior radiographs (upright position) by first drawing a line connecting the superior and inferior bony margins of the glenoid and then drawing a second line from the inferior bony margin of the glenoid to the most lateral border of the acromion [1] (Fig. 1). Slight malrotation in the coronal and sagittal plane was accepted since previous cadaver studies [1] showed constant CSA measurements in malrotation up to $20^{\circ}$.

\section{Measurements on CT scans}

All scans were made using a 64-slice CT unit (Toshiba, Torrance, CA, USA). Imaging was obtained in supine position with both arms in neutral rotation. Three-dimensional reconstructions of the scapula were computed using multiplanar reconstruction (MPR). The plane of measurement was systematically defined by the cut with the largest visible distance between the superior and inferior border of the glenoid on the coronal plane and the most lateral point on the cut with the most lateral extension of the acromion on the axial plane. In most of the cases, therefore, a new slight oblique coronal plane was defined by both readers independently since the standard coronal set did not include the most lateral extension of the acromion (Figs. 2 and 3). Clearly identifiable osteophytes were not included to angle measurements.

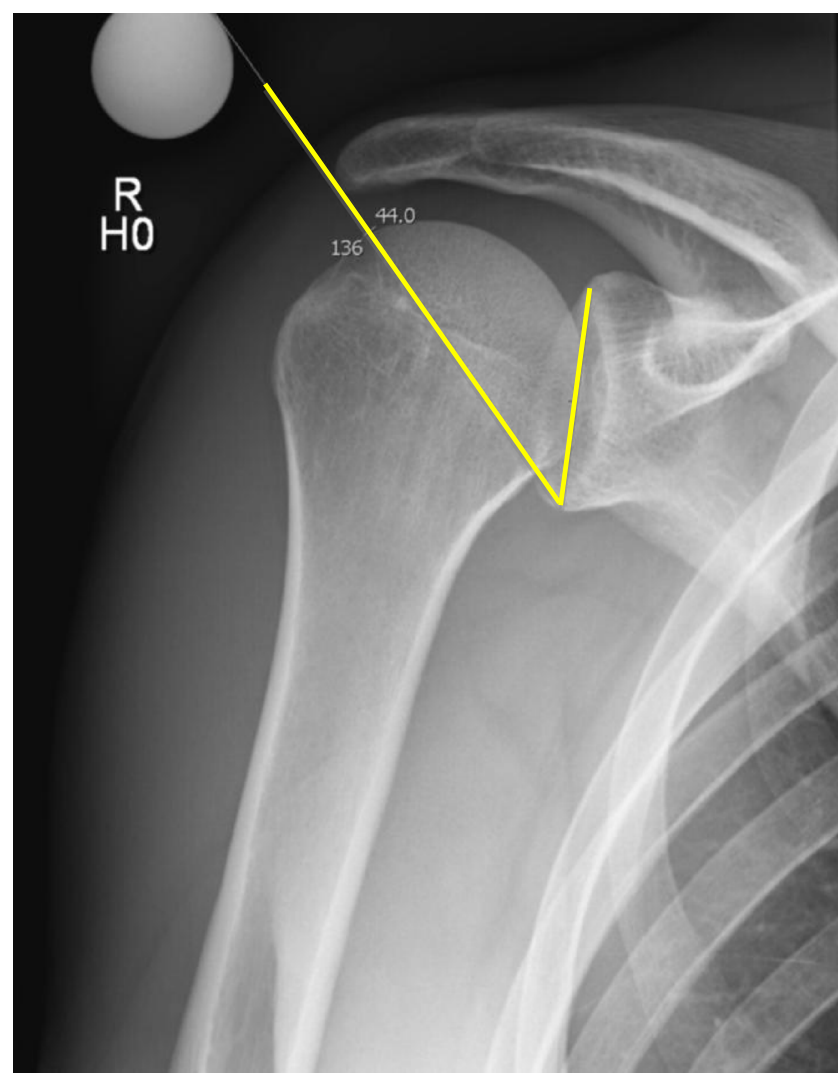

Fig. 1 Measurement of the critical shoulder angle on a conventional anterior-posterior radiograph of a right shoulder: One line is drawn to connect the superior and inferior bony margins of the glenoid, and a second line is drawn from the inferior bony margin of the glenoid to the most lateral border of the acromion

\section{Statistical analysis}

We expressed the distribution of variables using means and standard deviations for normally distributed data and medians and interquartile ranges for non-normally distributed data. We tested data for normality using the Kolmogorov-Smirnov test and performed quantile-quantile plots of dependent variables $[3,4]$.

We calculated inter-reader correlation coefficients (ICC) to assess the reproducibility of the ratings. We considered ICCs of 0.7 or higher to be sufficient [5]. We also calculated Spearman's correlation coefficient to assess the relationship between the CSA measured in the radiographs and CT scans.

All analyses were conducted using the STATA software (version 12, StataCorp., College Station, TX, USA).

\section{Results}

Of the 59 patients (60 shoulders) included in the study, 34 were male and 25 were female (median age, 60 years; interquartile range, 41.5-71 years). Glenohumeral osteoarthritis $(n=29)$ and shoulder instability $(n=16)$ were the most 


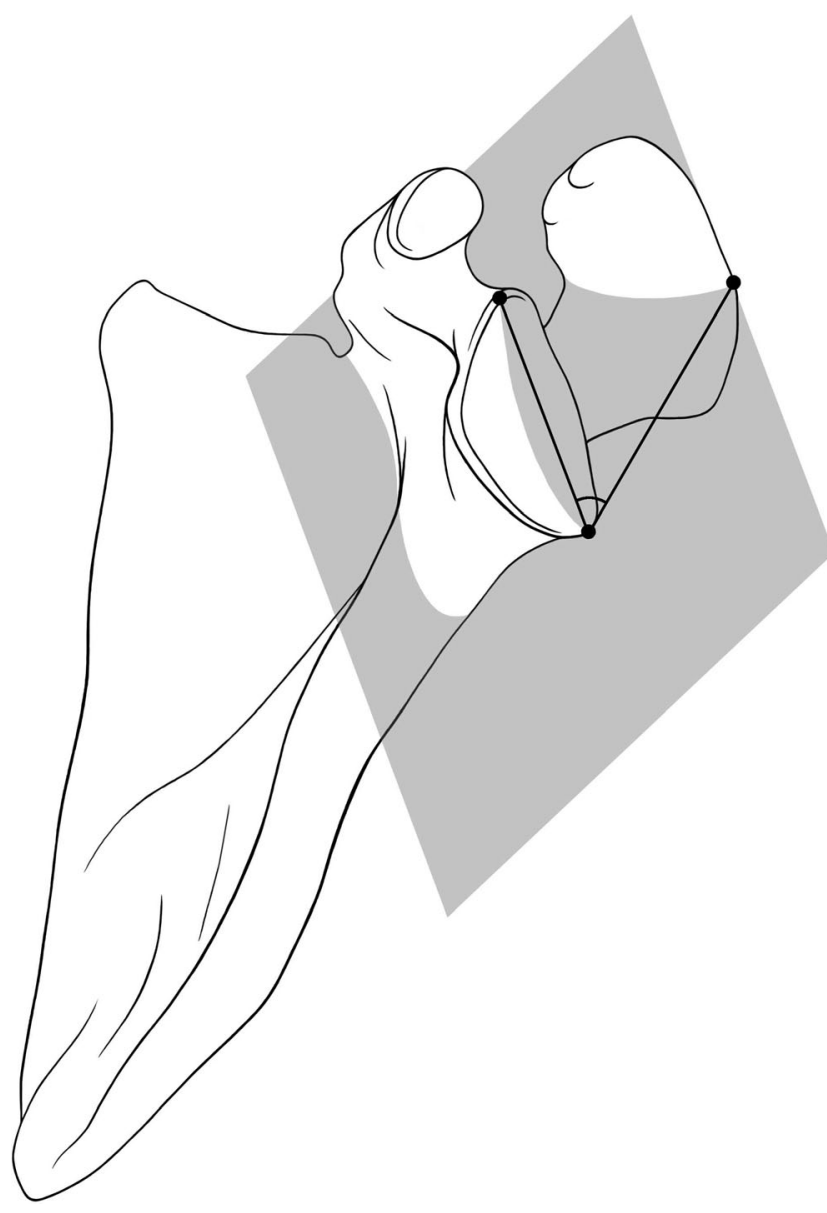

Fig. 2 Schematic view of the defined plane for the multiplanar reconstruction (MPR) of the CT scan. The plane is defined by the three points: superior border of the glenoid, inferior border of the glenoid and most lateral point of the acromial extension

frequent pathologies. Other diagnostic findings included unspecific shoulder pain $(n=5)$, rotator cuff tears $(n=4)$, rheumatoid arthritis $(n=3)$, one case with arthritis of the acromioclavicular joint, one subcapital humeral fracture and one fracture of the scapula without extension to the glenoid. A total of 30 left and 30 right shoulders were assessed.

The mean CSA measured by reader 1 was $33.2^{\circ}\left(\mathrm{SD} \pm 5.9^{\circ}\right)$ on radiographs and $33.3^{\circ}\left(\mathrm{SD} \pm 5.7^{\circ}\right)$ on $\mathrm{CT}$ scans, while the mean CSA measured by reader 2 was $33.1^{\circ}\left(\mathrm{SD} \pm 5.9^{\circ}\right)$ on radiographs and $33.0^{\circ}\left(\mathrm{SD} \pm 5.6^{\circ}\right)$ on $\mathrm{CT}$ scans. The mean discrepancy between reader 1 and reader 2 on radiographs was $0.2^{\circ}\left(\mathrm{SD} \pm 1.0^{\circ}\right)$, and the mean discrepancy on CT scan measurements was $0.3^{\circ}\left(\mathrm{SD} \pm 1.2^{\circ}\right)$.

The mean differences between radiographs and $\mathrm{CT}$ measurements were $-0.05^{\circ}\left(\mathrm{SD} \pm 1.2^{\circ}\right)$ for reader 1 and $0.1^{\circ}(\mathrm{SD}$ $\pm 1.2^{\circ}$ ) for reader 2 . The calculated inter-reader reliability was 0.993 for radiographs and 0.989 for CT scans. The intermethod correlation between the CSA measured on the radiographs and the CSA measured on CT scans was very strong $($ Spearman's rho=0.974) (Fig. 4).

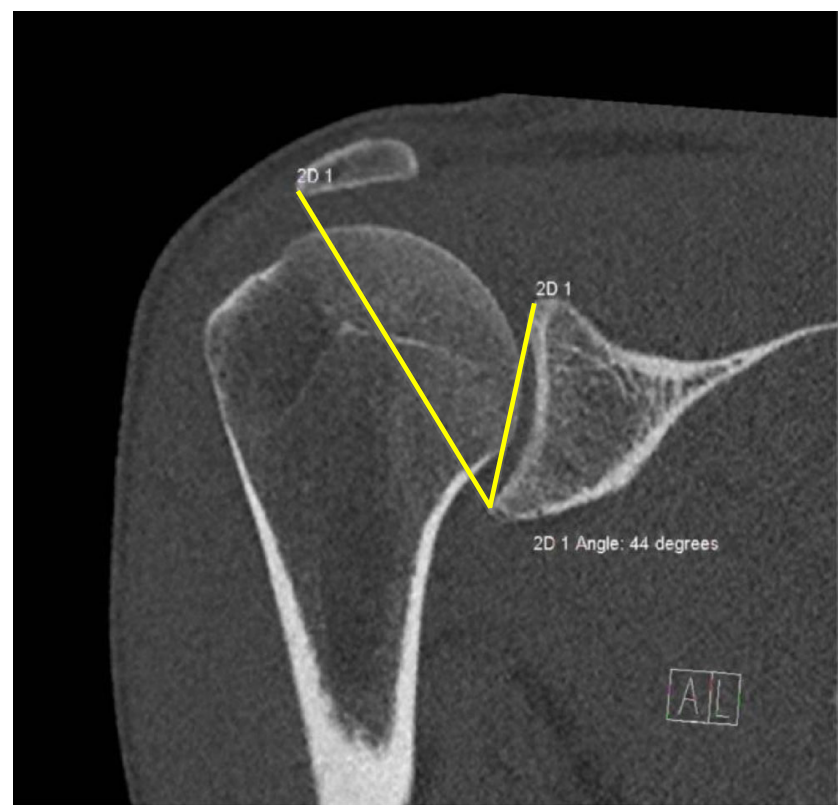

Fig. 3 Measurement of the critical shoulder angle on the defined plane with MPR on the corresponding CT scan of the same right shoulder. The angle is measured on the plane defined by the cut with the largest visible distance between the superior and inferior border of the glenoid on the coronal plane and the most lateral point on the cut with the most lateral extension of the acromion on the axial plane

\section{Discussion}

Combining the glenoid inclination and the lateral extension of the acromion in a single radiographic parameter, the CSA has proven to be reliable for the assessment of the scapular geometry associated with various degenerative shoulder pathologies $[1,2]$. Independent correlations between these two factors and shoulder impingement or degenerative cuff disease have previously been reported [6-9]. With the introduction of the CSA, a correlation between scapular geometry and glenohumeral osteoarthritis was demonstrated for the first

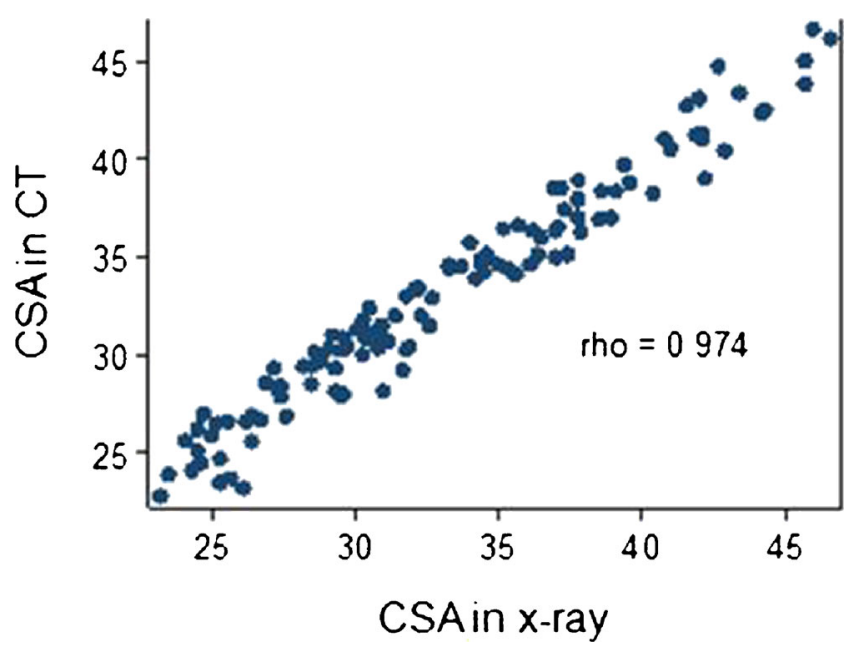

Fig. 4 Relationship between the CSAs measured on CT scans and radiographs 
time. While angles above $35^{\circ}$ are highly associated with degenerative cuff tears, measurements below $30^{\circ}$ are common in osteoarthritic shoulders [1]. Researchers have yet to prove the biomechanical concept of the "balanced shoulder" where mechanical loading by the deltoid muscle and its force vector promotes an increased load on the glenoid cartilage (low CSA) or on the supraspinatus tendon (high CSA) in unbalanced shoulders.

However, despite the reliability and reproducibility of the CSA measurement technique on radiographs, it is possible that angle measurements on cumulative images, such as radiographs, are not as precise as measurements on tomographic cross-sectional images where the exact landmarks can be defined. Because MRI images tend to display heterogenic definition of cartilage contours and bony edges, computed tomography, with its high resolution of bony structures, seems to be the most appropriate modality for precise CSA measurements. The option of computed reconstruction of the plane with the maximal superior-inferior distance of the glenoid combined with the exact determination of the coronal plane with the maximal lateral extent of the acromion is theoretically superior to measurements on three-dimensional, cumulative radiographs in terms of reproducibility and reliability.

The results of our study not only prove that measurements on CT scans are highly reliable but also indicate that the differences between measurements on anterior-posterior radiographs and CT scans are negligible. However, our data do not provide any explanation for the finding of radiographic and $\mathrm{CT}$ scan measurements being very similar despite the implementation of the third dimension with computed tomography. The relative position of the acromion to the glenoid in space has not been investigated in a large number of individuals yet. Further anatomical studies to clarify this issue are needed.

According to our results, CT-based assessments of the CSA are not necessary to improve measurement accuracy. Increased radiation exposure and costs can be avoided by using only radiographs.

The small number of patients (and shoulders) included is one of the study's limitations. However, the strong correlation of the measurements between radiographs and CT scans $(r=0.974)$ indicates sufficient cohort size. Furthermore, one of the two readers was a board-certified orthopaedic surgeon; therefore, potential differences in measurement technique compared to the fellowship-trained musculoskeletal radiologist may be postulated. This fact, we believe, has a minor impact only, since the orthopaedic surgeon is used to read CT scans of joints in his daily routine and was taught how to use the MPR software for this study.

\section{Conclusion}

The present data demonstrate that measurements of the CSA on anterior-posterior radiographs and CT scans are highly correlated and that inter-modality differences are negligible.

Conflict of interest The authors declare that they have no conflict of interest.

\section{References}

1. Moor BK, Bouaicha S, Rothenfluh DA, Sukthankar A, Gerber C. Is there an association between the individual anatomy of the scapula and the development of rotator cuff tears or osteoarthritis of the glenohumeral joint?: a radiological study of the critical shoulder angle. Bone Joint J. 2013;95-B(7):935-41.

2. Moor BK, Wieser K, Slankamenac K, Gerber C, Bouaicha S. Relationship of individual scapular anatomy and degenerative rotator cuff tears. J Shoulder Elbow Surg. 2014;23(4):536-41.

3. Solari M, Chakravarti I. Handbook of methods of applied statistics. Nature. 1967;216(5118):901-908.

4. Vardeman S. Graphical methods for data analysis. J Qual Technol. 1984;16(3):177-8.

5. Fleiss J. The design and analysis of clinical experiments. New York: Wiley; 1986.

6. Wong AS, Gallo L, Kuhn JE, Carpenter JE, Hughes RE. The effect of glenoid inclination on superior humeral head migration. J Shoulder Elbow Surg. 2003;12(4):360-4.

7. Hughes RE, Bryant CR, Hall JM, Wening J, Huston LJ, Kuhn JE, et al. Glenoid inclination is associated with full-thickness rotator cuff tears. Clin Orthop Relat Res. 2003;407:86-91.

8. Tetreault P, Krueger A, Zurakowski D, Gerber C. Glenoid version and rotator cuff tears. J Orthop Res. 2004;22(1):202-7.

9. Nyffeler RW, Werner CM, Sukthankar A, Schmid MR, Gerber C. Association of a large lateral extension of the acromion with rotator cuff tears. J Bone Joint Surg Am. 2006;88(4):800-5. 